Original Research Article

\title{
Evaluation of antioxidant potential of Emblica officinalis and Murraya koenigii and their role in modulation of cognitive function in diabetic rats
}

\author{
Tarun Arora ${ }^{1}$, Rudhra P. Kadali ${ }^{1}$, Dinesh K. Prasad ${ }^{2}$
}

Department of Pharmacology, ${ }^{1}$ Lady Hardinge Medical College, ${ }^{2}$ University College of Medical Sciences, New Delhi, India

Received: 25 June 2019

Revised: 16 August 2019

Accepted: 17 August 2019

*Correspondence to:

Dr. Rudhra P. Kadali,

Email: drrudhraprabhakar

@ gmail.com

Copyright: (C) the author(s), publisher and licensee Medip Academy. This is an openaccess article distributed under the terms of the Creative Commons Attribution NonCommercial License, which permits unrestricted noncommercial use, distribution, and reproduction in any medium, provided the original work is properly cited.

\section{ABSTRACT}

Background: Hyperglycaemia and hyperlipidaemia seen in diabetes mellitus result in oxidative stress and pose significant risk of cognitive decline that may lead to Alzheimer's disease. Approved anti-diabetic drugs have so far failed to demonstrate anti-oxidant and anti-hyperlipidemic activity, apart from saroglitazar. Therefore, this study was done to find a suitable anti-diabetic drug that possesses anti-hyperglycaemic, anti-oxidant and anti-hyperlipidemic activities and can reverse cognitive decline.

Methods: Emblica officinalis (250 mg/kg, p.o. and $500 \mathrm{mg} / \mathrm{kg}$, p.o.) and Murraya koenigii (250 mg/kg, p.o. and $500 \mathrm{mg} / \mathrm{kg}$, p.o.) were chosen to study these activities in Wistar rats. Diabetes was induced by single intraperitoneal injection of streptozotocin [STZ] $(50 \mathrm{mg} / \mathrm{kg})$. Fasting blood glucose levels and lipid profile were measured on day 1 and day 30 of the experiment. Cognitive function was assessed by measuring transfer latency (TL) on elevated plus maze, step-down latency (SDL) on passive avoidance apparatus and retention latency (RL) and quadrant time (QT) in Morris water maze. Oxidative stress was assessed at end of study by measuring brain MDA and GSH levels. Cholinergic marker of cognition, AChE was measured in brain at end of study.

Results: Both E. officinalis and M. koenigii showed dose dependent antihyperglycemic, anti-hyperlipidemic and anti-oxidant effects in diabetic rats with $500 \mathrm{mg} / \mathrm{kg}$ dose showing significantly higher effect. Both $250 \mathrm{mg} / \mathrm{kg}$ and 500 $\mathrm{mg} / \mathrm{kg}$ dose of E. officinalis and M. koenigii partially reversed cognitive decline in diabetic rats by day 30 .

Conclusions: $500 \mathrm{mg} / \mathrm{kg}$ p.o. dose of E. officinalis or M. koenigii has potential to reverse cognitive decline in diabetic patients.

Keywords: Emblica officinalis, Murraya koenigii, Oxidative stress, Cognitive decline. Diabetes

\section{INTRODUCTION}

Diabetes mellitus (DM), a complex syndrome is characterized primarily by imbalance in blood glucose homeostasis leading to hyperglycaemia (high blood sugar) and a series of secondary complications caused by an absolute or relative lack of insulin. Diabetes Mellitus is one of the most common endocrine disorders affecting almost $6 \%$ of the world's population. The number of diabetic patients will reach 300 million in 2025 (International Diabetic Federation, 2001). ${ }^{1}$
Despite progress in the management of diabetes mellitus by synthetic drugs most of these drugs have side effects in long run and drug resistance is also noted. ${ }^{2}$ So, the search for improved and safe natural antidiabetic agents is ongoing and World Health Organization has also recommended the development of herbal medicine in this concern. ${ }^{3}$ There is association of diabetes mellitus with cognitive dysfunction which is paralleled by neurophysiological and structural changes in $\mathrm{CNS}^{4}$

Emblica officinalis (Family - Euphorbiaceae), popularly known as Amla is one of the most celebrated herbs in 
Indian traditional medicine system, Ayurveda. Amla is highly nutritious and is an important dietary source of Vitamin C, minerals and amino acids. The fruit contains considerably higher concentration of most minerals and amino acids and has been reported to possess expectorant, purgative, spasmolytic, antibacterial, hypoglycemic, hepatoprotective and hypolipidemic activity. ${ }^{5-9}$

Murraya koenigii (syn. Bergera koenigii, Chalcas koenigii) is a tropical to sub-tropical plant belonging to family Rutaceae, which is native to India. It produces the leaves known as Curry leaves or Sweet Neem leaves. The hypoglycaemic effect of curry leaves has been studied in animal models. It has also been shown to possess antioxidant properties. ${ }^{10}$

As described above, both E. officinalis and M. koenigii possess antioxidant and hypoglycaemic properties. Considering the role of oxidative stress and the resultant cognitive impairment in diabetes, present study was done for evaluation of cognitive impairment and antioxidant potential of $E$. officinalis and $M$. koenigii in streptozotocin-induced diabetic rats.

\section{METHODS}

\section{Animals}

Wistar rats of either sex weighing between $150 \mathrm{~g}$ and 200 $\mathrm{g}$ were taken for the study. The rats were randomly distributed into different groups and were placed in polyvinyl cages. Each group contained ten rats, and the rats were numbered. The animals were housed under standard laboratory conditions and were fed with standard pelleted laboratory diet and water ad libitum. On the day of experiment rats were fasted overnight to avoid influence of food on drug absorption though water was given. Animals were maintained on a 12 hour natural light- dark cycle; temperature $22 \pm 1{ }^{\circ} \mathrm{C}$ and $50 \pm 2 \%$ relative humidity, with free access to food and water. The rats were acclimatized to laboratory conditions prior to experimentation.

Ethics committee approval was taken from the Institutional Animal Ethics Committee and the care of animals was done as per "CPCSEA Guidelines" for laboratory animal facilities.

\section{Drugs}

Distilled water, physiological saline, commercially available extract of fruit of $E$. officinalis, commercially available extract of leaves of $M$. koenigii, gliclazide, vitamin E, melatonin, atorvastatin.

\section{Chemicals}

Streptozotocin, other laboratory chemicals as will be required for estimation of biochemical parameters.

\section{Experimental design}

\section{Induction of diabetes}

Diabetes was induced by administering single intraperitoneal (i.p) injection of streptozotocin (50 $\mathrm{mg} / \mathrm{kg}$ ) dissolved in citrate buffer of $\mathrm{pH} 4.5$ in overnight fasted rats. The rats which had blood glucose levels $>200$ $\mathrm{mg} / \mathrm{dl}$ (fasting glycaemia) were considered as diabetic rats.

\section{Measurement of different parameters \\ Blood glucose and serum lipid profile}

Fasting blood samples were collected from the retroorbital plexus of the eye of rat and blood sugar was estimated by using glucose oxidase kit.

Fasting blood glucose levels were estimated till blood glucose levels stabilized i.e., BG >200 mg/dl and drug treatment was started from next day of blood glucose stabilization (which was taken as day 1) and continued for 30 days.

Blood samples were collected one day before the induction of diabetes and on 15th and 30th day after the stabilization of blood glucose levels for estimation of blood sugar and lipid profile (TC, LDL-c, HDL-c and TG).

\section{Cognitive assessment}

Behavioural tests to assess learning and memory were performed one day before the induction of diabetes and on 15 th and 30th day after the stabilization of blood glucose levels.

\section{Oxidative stress}

At the end of treatment, rats were sacrificed under ether anaesthesia and brain was taken out. Brain malondialdehyde, reduced glutathione and acetylcholinesterase levels were estimated.

In the experiment the rats were divided into following groups $(n=10)$ to study the antidiabetic and antioxidant effects of different doses of E. officinalis and M. koenigii and their role in modulation of cognitive dysfunction.

\section{Assessment of anti-hyperglycaemic and hypolipidemic activity}

- Group I Control (Distilled water): Vehicle of $E$. officinalis/ M. koenigii per oral (p.o).

- Group II Diabetic control: Streptozotocin $(50 \mathrm{mg} / \mathrm{kg}$, i.p).

- Group III Diabetic rats: E. officinalis per se; Dose I (250 mg/kg, p.o). 
- Group IV Diabetic rats: E. officinalis per se; Dose II (500 mg/kg, p.o).

- Group V Diabetic rats: M. koenigii per se; Dose I (250 mg/kg, p.o).

- Group VI Diabetic rats: $M$. koenigii per se; Dose II (500 mg/kg, p.o).

- Group VII Diabetic rats: Gliclazide (20 mg/kg, p.o).

- Group VIII Diabetic rats: Atorvastatin $(10 \mathrm{mg} / \mathrm{kg}$, p.o).

\section{Assessment of antioxidant activity and cognitive function}

Additional two groups of diabetic rats were taken for assessment of antioxidant activity and cognitive function as given below.

- Vitamin E+STZ: $100 \mathrm{mg} / \mathrm{kg}$, p.o./daily+50 mg/kg i.p.

- Melatonin+STZ: Melatonin $50 \mathrm{mg} / \mathrm{kg}$, p.o./daily+50 $\mathrm{mg} / \mathrm{kg}$ i.p.

Subsequently these groups were compared with the drug treated groups of E. officinalis and M. koenigii.

\section{Assessment of oxidative stress}

\section{Estimation of malondialdehyde}

Malondialdehyde (MDA) (indicator of lipid peroxidation) was estimated as per procedure described by Okhawa et al. $^{11}$

\section{Estimation of reduced glutathione}

Reduced glutathione (GSH) was estimated by the method as described by Ellman. ${ }^{12}$

\section{Estimation of brain acetylcholinesterase activity}

The rate of formation of thiocholine from acetylthiocholine iodide in the presence of tissue cholinesterase $(\mathrm{AChE})$ is measured by first treating with DTNB and then measuring the optical density (OD) of yellow coloured compound formed during the reaction at $412 \mathrm{~nm}$, every minute for a period of three minutes as per Ellman et al. ${ }^{13}$

\section{Assessment of cognition}

\section{Transfer latency on elevated plus maze}

Transfer latency (TL) was assessed through elevated plus maze test. TL is the time in which the animal moves from open arm to closed arm. The animal which does not enter a closed arm within $90 \mathrm{sec}$, were not included in the experiment. To become acquainted with the maze the animals were allowed to explore the maze for $20 \mathrm{~s}$ after reaching the closed arm and were then returned to their home cage. Retention was examined $24 \mathrm{~h}$ after the first day trial.

\section{Step down latency in continuous avoidance apparatus}

The rat was placed on the shock free zone (SFZ) and on stepping- down was given electric shock (20V) through the grid floor. The animal was returned to its cage and after $24 \mathrm{~h}$, the procedure was repeated and the time taken for the rat to step down was measured. This is known as step down latency (SDL). A prolongation of SDL was used as parameter of learning.

The animal groups were evaluated for TL and SDL one day before the induction of diabetes and on $14^{\text {th }}$ day and $29^{\text {th }}$ day after stabilization of blood glucose levels.

Morris water maze (Spatial navigation task)

The animal groups were evaluated for spatial navigation task on Morris water-maze one day before the induction of diabetes and on $15^{\text {th }}$ day and $30^{\text {th }}$ day after stabilization of blood glucose levels.

\section{Statistical analysis}

Results were analysed by one way Anova followed by post hoc Turkey's test for inter group comparison. Value of $p<0.05$ was taken as significant and value of $p<0.001$ was taken as highly significant.

\section{RESULTS}

Effect of various drugs (x30 days) on blood glucose levels

There was significant $(\mathrm{p}<0.001)$ increase in blood glucose levels in Diabetic control (II) group compared to nondiabetic control (I) group. At day '30' E. officinalis dose $250 \mathrm{mg} / \mathrm{kg}$ (III) group, Group IV, Group V, Group VI and Group VII showed "significant decrease" $(\mathrm{p}<0.001)$ in blood glucose levels when compared to Group II. Melatonin group $(50 \mathrm{mg} / \mathrm{kg})$, Vitamin E group (100 $\mathrm{mg} / \mathrm{kg}$ ) and group VIII did not show any significant change in blood glucose levels when compared to diabetic control group (Table 1).

\section{Effect of various drugs (x30 days) on serum total cholesterol levels}

Group II (diabetic control) showed "significant increase" $(\mathrm{p}<0.001)$ in total cholesterol levels at day ' 30 ' as compared to Group I. In comparison with Group II; the groups which showed "significant decrease" in total cholesterol levels were Group III $(\mathrm{p}<0.05)$, Group IV $(\mathrm{p}<0.01)$, Group V $(\mathrm{p}<0.05)$, Group VI $(\mathrm{p}<0.001)$ and Group VIII ( $\mathrm{p}<0.001)$. Melatonin group, Vitamin E group and Group VII did not show any significant change in total cholesterol level when compared to Group II (Table 2). 
Table 1: Effect of various drugs (x30 days) on blood glucose in STZ induced-diabetic rats (n=10).

\begin{tabular}{|c|c|c|c|}
\hline \multirow[t]{2}{*}{ Group } & \multirow{2}{*}{$\begin{array}{l}\text { Dose and route } \\
\text { (mg/kg, p.o.) }\end{array}$} & \multicolumn{2}{|c|}{$\begin{array}{l}\text { Blood glucose }(\mathrm{mg} / \mathrm{dl}) \\
\text { Mean } \pm \text { SEM }\end{array}$} \\
\hline & & Day 0 & Day 30 \\
\hline $\begin{array}{l}\text { Distilled water (non-diabetic } \\
\text { Control) }\end{array}$ & $\begin{array}{l}\text { Vehicle of E. officinalis and } \\
\text { M. koenigii }\end{array}$ & $74 \pm 4.61$ & $78.7 \pm 1.28$ \\
\hline Streptozotocin (diabetic control) & 50, i.p. & $291 \pm 5.22^{\mathrm{a}}$ & $298 \pm 4.36^{\mathrm{a}}$ \\
\hline $\begin{array}{l}\text { E. officinalis } \\
\text { Dose I+STZ }\end{array}$ & 250 & $278.2 \pm 3.27^{\mathrm{a}}$ & $166.7 \pm 2.44^{\mathrm{a}, \mathrm{b}}$ \\
\hline $\begin{array}{l}\text { E. officinalis } \\
\text { Dose II+STZ }\end{array}$ & 500 & $287.5 \pm 2.39^{\mathrm{a}}$ & $161.4 \pm 3.43^{\mathrm{a}, \mathrm{b}}$ \\
\hline $\begin{array}{l}\text { M. koenigii } \\
\text { Dose I+STZ }\end{array}$ & 250 & $281 \pm 3.23^{\mathrm{a}}$ & $188 \pm 2.67^{\mathrm{a}, \mathrm{b}}$ \\
\hline $\begin{array}{l}\text { M. koenigii } \\
\text { Dose II+STZ }\end{array}$ & 500 & $276 \pm 1.41^{\mathrm{a}, \mathrm{c}}$ & $152 \pm 1.22^{\mathrm{a}, \mathrm{b}}$ \\
\hline Melatonin+STZ & 50 & $272.2 \pm 2.42^{\mathrm{a}}$ & $289.6 \pm 2.24^{\mathrm{a}}$ \\
\hline Vitamin E+STZ & 100 & $275.7 \pm 3.41^{\mathrm{a}}$ & $294.1 \pm 1.43^{\mathrm{a}}$ \\
\hline Gliclazide+STZ & 20 & $281.7 \pm 2.43^{\mathrm{a}}$ & $164.9 \pm 1.62^{a, b}$ \\
\hline Atorvastatin+STZ & 10 & $288.2 \pm 1.41^{\mathrm{a}}$ & $290.6 \pm 2.11^{\mathrm{a}}$ \\
\hline
\end{tabular}

${ }_{\mathrm{a}}^{\mathrm{a}}<0.001$ as compared with non- diabetic control (distilled water) group; ${ }^{\mathrm{b}} \mathrm{p}<0.001$ as compared with diabetic control (STZ) group; c $\mathrm{p}<0.05$ as compared with diabetic control (STZ) group; One-way ANOVA followed by post-hoc Tukey’s test.

Table 2: Effect of various drugs (x30 days) on serum total cholesterol (TC), Triglycerides (TG), LDL-c and HDL-c levels in STZ induced-diabetic rats $(n=10)$.

\begin{tabular}{|c|c|c|c|c|c|}
\hline \multirow[t]{2}{*}{ Group } & \multirow[t]{2}{*}{$\begin{array}{l}\text { Dose and route } \\
\text { (mg/kg, p.o.) }\end{array}$} & \multicolumn{4}{|c|}{$\begin{array}{l}\text { Serum total cholesterol, triglycerides, LDL-c and HDL-c (mg/dl) } \\
\text { Mean } \pm \text { SEM }\end{array}$} \\
\hline & & Day 30 (TC) & Day 30 (TG) & Day 30 (LDL-c) & Day 30 (HDL-c) \\
\hline $\begin{array}{l}\text { Distilled water (non- } \\
\text { diabetic control) }\end{array}$ & $\begin{array}{l}\text { Vehicle of } \\
\text { E. officinalis and } \\
\text { M. koenigii }\end{array}$ & $135.2 \pm 3.74$ & $120.6 \pm 12.76$ & $65.4 \pm 3.74$ & $34 \pm 1.98$ \\
\hline $\begin{array}{l}\text { Streptozotocin } \\
\text { (diabetic control) }\end{array}$ & 50 , i.p. & $188.3 \pm 10.7^{\mathrm{a}}$ & $175.2 \pm 5.91^{\mathrm{d}}$ & $84.9 \pm 7.98$ & $23.6 \pm 3.76$ \\
\hline $\begin{array}{l}\text { E. officinalis } \\
\text { Dose I+STZ }\end{array}$ & 250 & $155.4 \pm 6.87^{\mathrm{d}}$ & $89.3 \pm 12.62^{\mathrm{a}}$ & $51.9 \pm 8.29^{\mathrm{d}}$ & $34.9 \pm 2.27$ \\
\hline $\begin{array}{l}\text { E. officinalis } \\
\text { Dose II+STZ }\end{array}$ & 500 & $145.1 \pm 3.49^{c}$ & $84 \pm 3.85^{\mathrm{a}}$ & $47.4 \pm 9.32^{c}$ & $41.1 \pm 4.08^{c}$ \\
\hline $\begin{array}{l}\text { M. koenigii } \\
\text { Dose I+STZ }\end{array}$ & 250 & $155.6 \pm 2.43^{\mathrm{d}}$ & $103.9 \pm 8.83^{\mathrm{a}}$ & $56.7 \pm 6.13$ & $40.2 \pm 4.72^{\mathrm{d}}$ \\
\hline $\begin{array}{l}\text { M. koenigii } \\
\text { Dose II+STZ }\end{array}$ & 500 & $140.7 \pm 7.34^{\mathrm{b}}$ & $81.1 \pm 11.08^{\mathrm{a}}$ & $51.2 \pm 4.67^{\mathrm{d}}$ & $44.2 \pm 2.12^{\mathrm{a}}$ \\
\hline Melatonin+STZ & 50 & $186.2 \pm 9.29^{\mathrm{a}}$ & $131.7 \pm 14.28$ & $78.7 \pm 4.65$ & $30.6 \pm 3.76$ \\
\hline Vitamin E+STZ & 100 & $180.1 \pm 5.43^{\mathrm{a}}$ & $138.2 \pm 16.87$ & $71.5 \pm 7.94$ & $32.5 \pm 1.87$ \\
\hline Gliclazide+STZ & 20 & $165.8 \pm 9.93$ & $124.5 \pm 12.8$ & $70.1 \pm 3.63$ & $33.6 \pm 3.22$ \\
\hline Atorvastatin+STZ & 10 & $130.3 \pm 1.87^{b}$ & $80.3 \pm 5.09^{a}$ & $48.3 \pm 4.47^{d}$ & $38.2 \pm 2.65^{\mathrm{d}}$ \\
\hline
\end{tabular}

${ }^{a} \mathrm{p}<0.001$ as compared with non-diabetic control (distilled water) group; ${ }^{b} \mathrm{p}<0.001$ as compared with diabetic control (STZ) group. ${ }^{c} \mathrm{p}<0.01$ as compared with diabetic control (STZ) group; ${ }^{\mathrm{d}} \mathrm{p}<0.05$ as compared with diabetic control (STZ) group; One-way ANOVA followed by post-hoc Tukey's test

\section{Effect of various drugs (x30 days) on serum total triglyceride levels}

At day ' 15 ', "significant increase" in total triglyceride was seen in Group II ( $\mathrm{p}<0.001)$ as compared to Group I. In comparison with Group II; the groups which showed "significant decrease" in total triglyceride levels at day ' 15 ' were E. officinalis dose $500 \mathrm{mg} / \mathrm{kg}$ (IV) group $(\mathrm{p}<0.001)$ and Group VIII $(\mathrm{p}<0.001)$.

At day '30', “significant increase" in total triglyceride was seen in Group II $(\mathrm{p}<0.05)$ as compared to Group I. In contrast to this, "significant decrease" in total triglyceride 
levels were seen in Group III, IV and VIII $(\mathrm{p}<0.01) M$. koenigii dose $250 \mathrm{mg} / \mathrm{kg}$ (V) group and M. koenigii dose $500 \mathrm{mg} / \mathrm{kg}$ (VI) group showed highest reduction in triglyceride levels $(\mathrm{p}<0.001)$ (Table 2).

\section{Effect of various drugs (x30 days) on serum total LDL- cholesterol levels}

At day ' 0 ' and ' 15 ' none of the studied compounds produced significant change in serum LDL-cholesterol levels when compared to Group II. At day '30'; the groups which showed "significant decrease" in serum LDL-cholesterol levels in comparison with Group II were Group III $(\mathrm{p}<0.05)$, Group IV $(\mathrm{p}<0.01)$, Group VI $(\mathrm{p}<0.05)$ and Group VIII $(\mathrm{p}<0.05)$ (Table 2$)$.
Effect of various drugs (x30 days) on serum HDLcholesterol levels

At day ' 30 '; the groups which showed "significant increase" in serum HDL-cholesterol levels in comparison with Group II were Group IV $(\mathrm{p}<0.01)$, Group V $(\mathrm{p}<0.05)$, Group VI $(\mathrm{p}<0.001)$ and Group VIII $(\mathrm{p}<0.05)$. (Table 2)

Effect of various drugs (x30 days) on behavioral parameters of learning and cognition

Acquisition of transfer latency (TL) in elevated plus-maze

No significant difference in TL was seen in any group at day ' 0 ' and day ' 14 ' as compared to Group I.

Table 3: Effect of various drugs (x30 days) on acquisition of transfer latency in STZ induced-diabetic rats (n=10).

\begin{tabular}{|c|c|c|c|c|}
\hline \multirow[t]{2}{*}{ Group } & \multirow{2}{*}{$\begin{array}{l}\text { Dose and route } \\
\text { (mg/kg, p.o.) }\end{array}$} & \multicolumn{3}{|c|}{$\begin{array}{l}\text { Transfer latency (seconds) } \\
\text { Mean } \pm \text { SEM }\end{array}$} \\
\hline & & Day 0 & Day 14 & Day 29 \\
\hline Distilled water (non-diabetic control) & $\begin{array}{l}\text { Vehicle of E. officinalis } \\
\text { and } M \text {. koenigii }\end{array}$ & $68.7 \pm 5.54$ & $59.4 \pm 7.23$ & $31.5 \pm 3.27$ \\
\hline Streptozotocin (diabetic control) & 50, i.p. & $86.6 \pm 6.78$ & $74.7 \pm 2.63$ & $64.7 \pm 4.07^{\mathrm{a}}$ \\
\hline $\begin{array}{l}\text { E. officinalis } \\
\text { Dose I+STZ }\end{array}$ & 250 & $81.4 \pm 4.67$ & $59.9 \pm 1.27$ & $51.6 \pm 5.28$ \\
\hline $\begin{array}{l}\text { E. officinalis } \\
\text { Dose II+STZ }\end{array}$ & 500 & $74.3 \pm 3.65$ & $53 \pm 5.76$ & $38.4 \pm 2.87^{\mathrm{d}}$ \\
\hline $\begin{array}{l}\text { M. koenigii } \\
\text { Dose I+STZ }\end{array}$ & 250 & $84.8 \pm 3.65$ & $62.1 \pm 3.88$ & $57.3 \pm 6.12^{b}$ \\
\hline $\begin{array}{l}\text { M. koenigii } \\
\text { Dose II+STZ }\end{array}$ & 500 & $76.4 \pm 2.05$ & $58.7 \pm 2.45$ & $43.7 \pm 4.41$ \\
\hline Melatonin+STZ & 50 & $69.4 \pm 4.76$ & $58.6 \pm 3.76$ & $46 \pm 6.55$ \\
\hline Vitamin E+STZ & 100 & $74.6 \pm 5.21$ & $67.6 \pm 4.67$ & $54.6 \pm 5.41^{\mathrm{c}}$ \\
\hline Gliclazide+STZ & 20 & $76.5 \pm 2.81$ & $65.9 \pm 2.56$ & $53.4 \pm 2.78$ \\
\hline Atorvastatin+STZ & 10 & $78.6 \pm 5.09$ & $61.7 \pm 2.77$ & $56.7 \pm 4.38^{\mathrm{b}}$ \\
\hline
\end{tabular}

${ }^{\mathrm{a}} \mathrm{p}<0.001$ as compared with non-diabetic control (distilled water) group; ${ }^{\mathrm{b}} \mathrm{p}<0.01$ as compared with non-diabetic control (distilled water) group; ${ }^{c} \mathrm{p}<0.05$ as compared with non-diabetic control (distilled water) group; ${ }^{\mathrm{d}} \mathrm{p}<0.01$ as compared with diabetic control (STZ) group. One-way ANOVA followed by post-hoc Tukey's test.

At day '29', "significant increase" in acquisition of TL was seen in Group II ( $\mathrm{p}<0.001)$ as compared to Group I. In comparison to Group II; the groups which showed "significant decrease" in acquisition of TL were Group IV $(\mathrm{p}<0.01)$, Group V $(\mathrm{p}<0.01)$, Vitamin E group $(\mathrm{p}<0.05)$ and Group VIII $(\mathrm{p}<0.01)$ (Table 3$)$.

\section{Retention of transfer latency $(T L)$ in elevated plus-maze}

No significant difference was seen in any group at day ' 1 ' and day ' 15 ' as compared to Group I. At day 30, "significant increase" in retention of TL was seen in Group II $(\mathrm{p}<0.01)$ as compared to Group I.
"Significant decrease" in retention of TL was seen in Group IV $(\mathrm{p}<0.01)$, Group VI $(\mathrm{p}<0.01)$, and melatonin group ( $<<0.001)$ as compared to Group II (Table 4).

Acquisition of step-down latency (SDL) in continuous avoidance apparatus

At day '14" "significant decrease" in acquisition of SDL was seen in Group II $(\mathrm{p}<0.01)$ as compared to Group I and "significant increase" in acquisition of SDL was seen in Group III $(\mathrm{p}<0.01)$, Melatonin group $(\mathrm{p}<0.05)$ and Group VII $(\mathrm{p}<0.01)$ as compared to Group II. 
Table 4: Effect of various drugs (x30 days) on retention of transfer latency in STZ induced-diabetic rats (n=10).

\begin{tabular}{|c|c|c|c|c|}
\hline \multirow[t]{2}{*}{ Group } & \multirow{2}{*}{ Dose and route (mg/kg, p.o.) } & \multicolumn{3}{|c|}{$\begin{array}{l}\text { Transfer latency (seconds) } \\
\text { Mean } \pm \text { SEM }\end{array}$} \\
\hline & & Day 1 & Day 15 & Day 30 \\
\hline $\begin{array}{l}\text { Distilled water (non- } \\
\text { diabetic control) }\end{array}$ & $\begin{array}{l}\text { Vehicle of } \text { E. officinalis and } \\
\text { M. koenigii }\end{array}$ & $62 \pm 6.94$ & $36.8 \pm 5.87$ & $27.6 \pm 1.74$ \\
\hline $\begin{array}{l}\text { Streptozotocin } \\
\text { (diabetic control) }\end{array}$ & 50, i.p. & $67.8 \pm 3.56$ & $59.3 \pm 4.87$ & $56.4 \pm 4.93^{\mathrm{a}}$ \\
\hline $\begin{array}{l}\text { E. officinalis } \\
\text { Dose I+STZ }\end{array}$ & 250 & $62.4 \pm 5.57$ & $53.7 \pm 7.11$ & $36.9 \pm 2.94$ \\
\hline $\begin{array}{l}\text { E. officinalis } \\
\text { Dose II+STZ }\end{array}$ & 500 & $59.5 \pm 4.93$ & $44 \pm 5.98$ & $30 \pm 4.08^{c}$ \\
\hline $\begin{array}{l}\text { M. koenigii } \\
\text { Dose I+STZ }\end{array}$ & 250 & $71.6 \pm 6.44$ & $54 \pm 3.27$ & $42 \pm 4.45$ \\
\hline $\begin{array}{l}\text { M. koenigii } \\
\text { Dose II+STZ }\end{array}$ & 500 & $68 \pm 4.65$ & $56 \pm 6.23$ & $29 \pm 7.34^{c}$ \\
\hline Melatonin+STZ & 50 & $64.6 \pm 3.68$ & $48.8 \pm 5.22$ & $20.7 \pm 8.19^{b}$ \\
\hline Vitamin E+STZ & 100 & $66.3 \pm 6.23$ & $54.3 \pm 5.79$ & $43.5 \pm 4.74$ \\
\hline Gliclazide+STZ & 20 & $72.2 \pm 4.62$ & $56.6 \pm 6.24$ & $45.7 \pm 3.93$ \\
\hline Atorvastatin+STZ & 10 & $69 \pm 2.76$ & $52 \pm 4.66$ & $49 \pm 2.93$ \\
\hline
\end{tabular}

${ }^{\mathrm{a}} \mathrm{p}<0.01$ as compared with non-diabetic control (distilled water) group; ${ }^{\mathrm{b}} \mathrm{p}<0.001$ as compared with diabetic control (STZ) group; c $\mathrm{p}<0.01$ as compared with diabetic control (STZ) group; One-way ANOVA followed by post-hoc Tukey’s test.

Table 5: Effect of various drugs (X 30 days) on acquisition of step-down latency in STZ induced-diabetic rats $(n=10)$.

\begin{tabular}{|c|c|c|c|c|}
\hline \multirow[t]{2}{*}{ Group } & \multirow[t]{2}{*}{ Dose \& Route (mg/kg, p.o.) } & \multicolumn{3}{|c|}{$\begin{array}{l}\text { Step-down latency (seconds) } \\
\text { Mean } \pm \text { SEM }\end{array}$} \\
\hline & & Day 0 & Day 14 & Day 29 \\
\hline $\begin{array}{l}\text { Distilled water (non- } \\
\text { diabetic control) }\end{array}$ & $\begin{array}{l}\text { Vehicle of E. officinalis and } \\
\text { M. koenigii }\end{array}$ & $182.3 \pm 12.56$ & $235.7 \pm 7.43$ & $274.2 \pm 3.93$ \\
\hline $\begin{array}{l}\text { Streptozotocin } \\
\text { (diabetic control) }\end{array}$ & 50, i.p. & $171.7 \pm 12.63$ & $169.7 \pm 14.98^{\mathrm{a}}$ & $167.4 \pm 12.96^{\mathrm{a}}$ \\
\hline $\begin{array}{l}\text { E. officinalis } \\
\text { Dose I+STZ }\end{array}$ & 250 & $182.7 \pm 11.45$ & $237.1 \pm 5.98^{\mathrm{d}}$ & $250.2 \pm 14.16^{\mathrm{c}}$ \\
\hline $\begin{array}{l}\text { E. officinalis } \\
\text { Dose II+STZ }\end{array}$ & 500 & $178.5 \pm 11.64$ & $202.5 \pm 10.43$ & $242.8 \pm 12.43^{c}$ \\
\hline $\begin{array}{l}\text { M. koenigii } \\
\text { Dose I+STZ }\end{array}$ & 250 & $170.5 \pm 12.44$ & $192.16 \pm 9.44$ & $230 \pm 10.56^{\mathrm{d}}$ \\
\hline $\begin{array}{l}\text { M. koenigii } \\
\text { Dose II+STZ }\end{array}$ & 500 & $181 \pm 14.78$ & $204.7 \pm 15.98$ & $244.4 \pm 12.87^{\mathrm{c}}$ \\
\hline Melatonin+STZ & 50 & $185.8 \pm 14.56$ & $224.2 \pm 14.11^{\mathrm{e}}$ & $243.2 \pm 12.43^{\mathrm{c}}$ \\
\hline Vitamin E+STZ & 100 & $172.4 \pm 12.82$ & $198 \pm 11.16$ & $214 \pm 17.45^{b}$ \\
\hline Gliclazide+STZ & 20 & $191.5 \pm 12.97$ & $232.5 \pm 10.66^{\mathrm{d}}$ & $247.9 \pm 6.87^{\mathrm{c}}$ \\
\hline Atorvastatin+STZ & 10 & $188.5 \pm 4.87$ & $201.6 \pm 9.54$ & $226.3 \pm 4.23^{\mathrm{e}}$ \\
\hline
\end{tabular}

${ }^{a} \mathrm{p}<0.01$ as compared with non-diabetic control (distilled water) group; ${ }^{b} \mathrm{p}<0.05$ as compared with non-diabetic control (distilled water) group; ${ }^{\mathrm{c}} \mathrm{p}<0.001$ as compared with diabetic control (STZ) group; ${ }^{\mathrm{d}} \mathrm{p}<0.01$ as compared with diabetic control (STZ) group; ${ }^{\mathrm{e}} \mathrm{p}<0.05$ as compared with diabetic control (STZ) group; One-way ANOVA followed by post-hoc Tukey's test.

At day '29', "significant decrease" in acquisition of SDL was seen in Group II $(\mathrm{p}<0.01)$ as compared to Group I. In comparison with Group II; the groups which showed "significant increase" in acquisition of SDL are Group III and IV $(p<0.001)$, Group V $(p<0.01)$, Group VI $(\mathrm{p}<0.001)$, Melatonin group $(\mathrm{p}<0.001)$, Group VII $(\mathrm{p}<0.001)$ and Group VIII $(\mathrm{p}<0.05)$.

However, Vitamin E group did not show any significant change in acquisition of TL when compared to Group II (Table 5). 
Table 6: Effect of various drugs (x30 days) on retention of step-down latency in Streptozotocin (STZ) induceddiabetic rats $(n=10)$.

\begin{tabular}{|c|c|c|c|c|}
\hline \multirow[t]{2}{*}{ Group } & \multirow{2}{*}{$\begin{array}{l}\text { Dose and route (mg/kg, } \\
\text { p.o.) }\end{array}$} & \multicolumn{3}{|c|}{$\begin{array}{l}\text { Step-down latency (Seconds) } \\
\text { Mean } \pm \text { SEM }\end{array}$} \\
\hline & & Day 1 & Day 15 & Day 30 \\
\hline $\begin{array}{l}\text { Distilled water (Non-diabetic } \\
\text { Control) }\end{array}$ & $\begin{array}{l}\text { Vehicle of } E \text {. officinalis } \\
\text { and } M \text {. koenigii }\end{array}$ & $212.4 \pm 13.69$ & $236.7 \pm 12.76$ & $261 \pm 3.94$ \\
\hline Streptozotocin (Diabetic Control) & 50, i.p. & $196.54 \pm 16.7$ & $188.5 \pm 15.44^{\mathrm{a}}$ & $190.3 \pm 18.87^{\mathrm{a}}$ \\
\hline E. officinalis Dose I+STZ & 250 & $210.6 \pm 14.87$ & $226.7 \pm 9.93$ & $251.6 \pm 5.88^{d}$ \\
\hline E. officinalis Dose II+STZ & 500 & $221.7 \pm 21.66$ & $257 \pm 10.43^{b}$ & $283.8 \pm 16.77^{\mathrm{b}}$ \\
\hline M. koenigii Dose I+STZ & 250 & $219.6 \pm 6.65$ & $221.7 \pm 9.66$ & $249 \pm 13.93$ \\
\hline M. koenigii Dose II+STZ & 500 & $208.7 \pm 12.76$ & $216.7 \pm 7.98$ & $252.7 \pm 19.76^{d}$ \\
\hline Melatonin+STZ & 50 & $212 \pm 12.46$ & $234.6 \pm 7.66$ & $252.8 \pm 11.63^{\mathrm{d}}$ \\
\hline Vitamin E+STZ & 100 & $228.4 \pm 13.34$ & $229 \pm 12.47$ & $264.7 \pm 9.88^{c}$ \\
\hline Gliclazide+STZ & 20 & $213.5 \pm 13.28$ & $235.2 \pm 5.43$ & $264.9 \pm 7.86^{\mathrm{c}}$ \\
\hline Atorvastatin+STZ & 10 & $227 \pm 12.22$ & $232.6 \pm 7.84$ & $258.7 \pm 13.87^{\mathrm{d}}$ \\
\hline
\end{tabular}

Table 7: Effect of various drugs(x30 days) on retention of spatial navigation task in STZ induced-diabetic rats $(\mathbf{n}=10)$.

\begin{tabular}{|c|c|c|c|c|}
\hline \multirow{2}{*}{ Group } & \multirow{2}{*}{ Treatment and route } & \multicolumn{3}{|c|}{$\begin{array}{l}\text { Latency (seconds) } \\
\text { Mean } \pm \text { SEM }\end{array}$} \\
\hline & & Day 1; IAL & Day 15; RL-1 & Day 30; RL-2 \\
\hline Distilled water (Non-diabetic Control) & $\begin{array}{l}\text { Vehicle of E. officinalis } \\
\text { and } M . \text { koenigii }\end{array}$ & $40.4 \pm 7.54$ & $38.7 \pm 5.12$ & $48.2 \pm 3.65$ \\
\hline Streptozotocin (Diabetic Control) & 50, i.p. & $49.2 \pm 2.63$ & $72.4 \pm 3.64$ & $95.6 \pm 4.32^{\mathrm{a}}$ \\
\hline E. officinalis Dose I+STZ & 250 & $59.8 \pm 2.17$ & $48.1 \pm 4.65$ & $45.3 \pm 2.71^{\mathrm{c}}$ \\
\hline E. officinalis Dose II+STZ & 500 & $64.5 \pm 2.71$ & $51.4 \pm 3.97$ & $42.9 \pm 4.17^{\mathrm{c}}$ \\
\hline M. koenigii Dose I+STZ & 250 & $51.3 \pm 2.72$ & $44.6 \pm 2.12$ & $35.9 \pm 3.54^{\mathrm{c}}$ \\
\hline M. koenigii Dose II+STZ & 500 & $63.3 \pm 2.19$ & $59.6 \pm 6.72$ & $43.1 \pm 3.48^{\mathrm{c}}$ \\
\hline Melatonin+STZ & 50 & $63.3 \pm 2.64$ & $57 \pm 3.66$ & $54.6 \pm 3.56^{\mathrm{c}}$ \\
\hline Vitamin E+STZ & 100 & $61.7 \pm 3.44$ & $54.9 \pm 4.74$ & $44.5 \pm 2.62^{c}$ \\
\hline Gliclazide+STZ & 20 & $68.1 \pm 2.41$ & $53.6 \pm 4.42$ & $49.3 \pm 4.21^{\mathrm{c}}$ \\
\hline Atorvastatin+STZ & 10 & $54.2 \pm 3.34$ & $45.6 \pm 2.56$ & $29.3 \pm 3.54^{\mathrm{b}, \mathrm{c}}$ \\
\hline
\end{tabular}

${ }^{\mathrm{a}} \mathrm{p}<0.001$ as compared with non-diabetic control (Distilled water) group, ${ }^{\mathrm{b}} \mathrm{p}<0.05$ as compared with non-diabetic control (Distilled water) group, ${ }^{\mathrm{c}} \mathrm{p}<0.001$ as compared with diabetic control (STZ) group, One-way ANOVA followed by post-hoc Tukey's test.

Table 8: Effect of various drugs (x30 days) on 'Quadrant-time' of spatial navigation task in Streptozotocin (STZ) induced-diabetic rats $(\mathrm{n}=10)$.

\begin{tabular}{|c|c|c|c|c|}
\hline \multirow{2}{*}{ Group } & \multirow{2}{*}{$\begin{array}{l}\text { Dose and route (mg/kg, } \\
\text { p.o.) }\end{array}$} & \multicolumn{3}{|c|}{$\begin{array}{l}\text { Time (Seconds) } \\
\text { Mean } \pm \text { SEM }\end{array}$} \\
\hline & & Day 1; IAL & Day15;QT-1 & Day 30;QT-2 \\
\hline Distilled water (non-diabetic control) & $\begin{array}{l}\text { Vehicle of E. officinalis } \\
\text { and } M . \text { koenigii }\end{array}$ & $43 \pm 4.67$ & $61 \pm 4.44$ & $69.4 \pm 4.51$ \\
\hline Streptozotocin (diabetic control) & 50, i.p. & $55.5 \pm 2.76$ & $34.2 \pm 3.43^{\mathrm{a}}$ & $19.5 \pm 3.14^{\mathrm{a}}$ \\
\hline E. officinalis Dose I+STZ & 250 & $59.6 \pm 2.65$ & $41.7 \pm 3.97$ & $48.2 \pm 4.73^{\mathrm{a}, \mathrm{d}}$ \\
\hline E. officinalis Dose II+STZ & 500 & $65.3 \pm 3.23$ & $43.8 \pm 3.7^{\mathrm{c}}$ & $55 \pm 3.26^{\mathrm{a}, \mathrm{d}}$ \\
\hline M. koenigii Dose I+STZ & 250 & $52.6 \pm 2.65$ & $38.6 \pm 3.54^{\mathrm{a}}$ & $43.3 \pm 2.74^{\mathrm{a}, \mathrm{d}}$ \\
\hline M. koenigii Dose II+STZ & 500 & $54.4 \pm 3.12$ & $41.3 \pm 2.17^{\mathrm{c}}$ & $48.3 \pm 2.28^{d}$ \\
\hline Melatonin+STZ & 50 & $58.3 \pm 3.26$ & $39.7 \pm 2.71^{b}$ & $66.2 \pm 3.21^{\mathrm{d}}$ \\
\hline Vitamin E+STZ & 100 & $49.5 \pm 2.23$ & $42.6 \pm 2.33^{\mathrm{c}}$ & $34 \pm 3.04^{\mathrm{a}}$ \\
\hline Gliclazide+STZ & 20 & $51.6 \pm 3.58$ & $41.1 \pm 3.09^{b}$ & $42.1 \pm 2.56^{\mathrm{a}, \mathrm{b}}$ \\
\hline Atorvastatin+STZ & 10 & $56.3 \pm 2.32$ & $40.6 \pm 4.03$ & $44.5 \pm 2.19^{\mathrm{a}, \mathrm{b}}$ \\
\hline
\end{tabular}


Table 9: Effect of various drugs (x30 days) on brain levels of MDA and GSH in Streptozotocin (STZ) induced Diabetic rats $(\mathbf{n}=\mathbf{1 0})$.

\begin{tabular}{|c|c|c|c|}
\hline Group & $\begin{array}{l}\text { Dose and route }(\mathrm{mg} / \mathrm{kg} \text {, } \\
\text { p.o.) }\end{array}$ & $\begin{array}{l}\text { MDA (nmol/g } \\
\text { wet brain tissue) } \\
\text { Mean } \pm \text { SEM }\end{array}$ & $\begin{array}{l}\text { GSH ( } \mu g / g \text { wet } \\
\text { brain tissue) } \\
\text { Mean } \pm \text { SEM }\end{array}$ \\
\hline Distilled water (Non-diabetic Control) & $\begin{array}{l}\text { Vehicle of E. officinalis } \\
\text { and } M \text {. koenigii }\end{array}$ & $191.4 \pm 12.45$ & $364.3 \pm 11.76$ \\
\hline Streptozotocin (Diabetic Control) & 50, i.p. & $346.8 \pm 16.76^{\mathrm{a}}$ & $192.4 \pm 14.38^{\mathrm{a}}$ \\
\hline $\begin{array}{l}\text { E. officinalis } \\
\text { Dose I+STZ }\end{array}$ & 250 & $252.4 \pm 15.88^{c}$ & $296.3 \pm 16.71^{\mathrm{c}}$ \\
\hline $\begin{array}{l}\text { E. officinalis } \\
\text { Dose II+STZ }\end{array}$ & 500 & $234.2 \pm 12.32^{\mathrm{c}}$ & $322.7 \pm 11.14^{\mathrm{c}}$ \\
\hline $\begin{array}{l}\text { M. koenigii } \\
\text { Dose I+STZ }\end{array}$ & 250 & $265.8 \pm 10.76^{\mathrm{b}, \mathrm{d}}$ & $301.4 \pm 13.61$ \\
\hline $\begin{array}{l}\text { M. koenigii } \\
\text { Dose II+STZ }\end{array}$ & 500 & $241.3 \pm 13.33^{c}$ & $312 \pm 12.79^{c}$ \\
\hline Melatonin+STZ & 50 & $220.6 \pm 13.34^{c}$ & $259.3 \pm 6.03^{\mathrm{a}}$ \\
\hline Vitamin E+STZ & 100 & $191.7 \pm 12.45^{\mathrm{c}}$ & $334.3 \pm 11.54^{\mathrm{c}}$ \\
\hline Gliclazide+STZ & 20 & $246.3 \pm 11.92^{c}$ & $241.2 \pm 7.65^{\mathrm{a}}$ \\
\hline Atorvastatin+STZ & 10 & $284.7 \pm 14.43^{\mathrm{a}}$ & $252.6 \pm 12.39^{\mathrm{a}, \mathrm{e}}$ \\
\hline
\end{tabular}

${ }^{a} \mathrm{p}<0.001$ as compared with non-diabetic control (Distilled water) group, ${ }^{b} \mathrm{p}<0.01$ as compared with non-diabetic control (Distilled water) group, ${ }^{c} \mathrm{p}<0.001$ as compared with diabetic control (STZ) group, ${ }^{\mathrm{d}} \mathrm{p}<0.01$ as compared with diabetic control (STZ) group, ${ }^{\mathrm{e}} \mathrm{p}<0.05$ as compared with diabetic control (STZ) group, One-way ANOVA followed by post-hoc Tukey’s test

Table 10: Effect of various drugs (x30 days) on brain levels of AChE in Streptozotocin (STZ) induced-diabetic rats $(\mathbf{n}=\mathbf{1 0})$.

\begin{tabular}{|lll|}
\hline Group & Treatment and route & $\begin{array}{l}\text { AChE activity (mol/min/g protein) } \\
\text { Mean } \pm \text { SEM }\end{array}$ \\
\hline Distilled water (non-diabetic control) & $\begin{array}{l}\text { Vehicle of } \text { E. officinalis and } \\
\text { M. koenigii }\end{array}$ & $162.2 \pm 13.65$ \\
\hline $\begin{array}{l}\text { Streptozotocin } \\
\text { diabetic control) }\end{array}$ & 50, i.p. & $267.3 \pm 12.29^{\mathrm{a}}$ \\
\hline $\begin{array}{l}\text { E. } \text { officinalis } \\
\text { Dose I+STZ }\end{array}$ & 250 & $209.5 \pm 5.76^{\mathrm{a}, \mathrm{d}}$ \\
\hline $\begin{array}{l}\text { E. officinalis } \\
\text { Dose II+STZ }\end{array}$ & 500 & $196.1 \pm 12.43^{\mathrm{a}, \mathrm{c}}$ \\
\hline $\begin{array}{l}\text { M. } \text { koenigii } \\
\text { Dose I+STZ }\end{array}$ & 250 & $216.7 \pm 14.56^{\mathrm{a}}$ \\
\hline $\begin{array}{l}\text { M. } \text { koenigii } \\
\text { Dose II+STZ }\end{array}$ & 500 & $197.4 \pm 12.71^{\mathrm{a}, \mathrm{c}}$ \\
\hline Melatonin+STZ & 50 & $184.3 \pm 10.32^{\mathrm{a}, \mathrm{b}}$ \\
\hline Vitamin E+STZ & 100 & $226 \pm 13.22^{\mathrm{a}}$ \\
\hline Gliclazide+STZ & 20 & $238.7 \pm 11.54^{\mathrm{a}}$ \\
\hline Atorvastatin+STZ & 10 & $242.1 \pm 13.67^{\mathrm{a}}$ \\
\hline
\end{tabular}

${ }^{\mathrm{a}} \mathrm{p}<0.001$ as compared with non-diabetic control (distilled water) group, ${ }^{\mathrm{b}} \mathrm{p}<0.001$ as compared with diabetic control (STZ) group, ${ }^{c} \mathrm{p}<0.01$ as compared with diabetic control (STZ) group, ${ }^{\mathrm{d}} \mathrm{p}<0.05$ as compared with diabetic control (STZ) group, One-way ANOVA followed by post-hoc Tukey's test.

Retention of step-down latency (SDL) in continuous avoidance apparatus

At day '15', "significant decrease" in retention of SDL was seen in diabetic control group II $(\mathrm{p}<0.05)$ as compared to group I. Group IV $(\mathrm{p}<0.001)$ showed "significant increase" in retention of SDL as compared to Group II.
At day '30', "significant decrease" in retention of SDL was seen in Group II $(\mathrm{p}<0.05)$ when compared to Group I. In comparison with Group II; the groups which showed "significant increase" in retention of SDL are Group III $(\mathrm{p}<0.05)$, Group IV $(\mathrm{p}<0.001)$, Group VI $(\mathrm{p}<0.05)$, Melatonin group $(\mathrm{p}<0.05)$, Vitamin $\mathrm{E}$ group $(\mathrm{p}<0.01)$, Group VII $(\mathrm{p}<0.01)$, Group VIII $(\mathrm{p}<0.05)$ (Table 6). 
Retention of spatial navigation task in Morris Water Maze

No significant difference was seen in IAL in any group at day ' 1 and ' 15 ' compared to Group I.

At day '30', "significant increase" in RL-2 was seen in Group II $(\mathrm{p}<0.001)$ as compared to Group I. In comparison with Group II; the groups which showed "significant decrease" in RL-2 were Group III and IV $(\mathrm{p}<0.001)$, Group V and VI $(\mathrm{p}<0.05)$, Melatonin group $(p<0.001)$, Vitamin E group $(p<0.001)$, Group VII $(\mathrm{p}<0.001)$, Group III $(\mathrm{p}<0.001)$ (Table 7).

'Quadrant-time' (QT) in spatial navigation task in Morris Water Maze

No significant difference was seen in any group in IAL at day ' 1 compared to No treatment group 'I'.

At day '15', "significant decrease" in QT-1 was seen in Group II ( $\mathrm{p}<0.001)$ as compared to Group I.

At day '30', "significant decrease" in QT-2 was seen in Group II $(\mathrm{p}<0.001)$ as compared to Group I. In comparison "significant increase" in QT-2 were seen in Group III and IV $(\mathrm{p}<0.001)$, Group V and VI $(\mathrm{p}<0.001)$ and Melatonin group $(\mathrm{p}<0.001)$ (Table 8).

\section{Effect of various drugs (x30 day) on biochemical parameters}

\section{MDA and GSH levels in brain}

In streptozotocin (STZ) induced diabetic group II, "significant increase" in brain MDA levels were seen $(\mathrm{p}<0.001)$ as compared to Group I. In comparison to Group II; the groups which showed "significant decrease" in brain MDA levels were - Group III and IV $(\mathrm{p}<0.001)$, Group V $(\mathrm{p}<0.05)$, Group VI $(\mathrm{p}<0.001)$, Melatonin group $(p<0.001)$, Vitamin E group $(p<0.001)$ and Group VII $(\mathrm{p}<0.001)$.

"Significant decrease" in brain GSH levels among diabetic rats was seen in Group II $(\mathrm{p}<0.001)$ as compared to Group I. In comparison to Group II; the groups which showed "significant increase" in brain GSH levels were Group III and IV $(p<0.001)$, Group VI $(p<0.001)$, Vitamin E group $(\mathrm{p}<0.001)$ and Group VIII $(\mathrm{p}<0.05)$ (Table 9).

\section{AChE levels in Brain}

Among streptozotocin (STZ) induced diabetic group II, brain AChE levels were found to be "significantly increased" ( $p<0.001)$ as compared to Group I. Group III $(\mathrm{p}<0.05)$, Group IV $(\mathrm{p}<0.01)$ and Group VI $(\mathrm{p}<0.01)$ showed "significant decrease" in brain AChE levels as compared to group II.
However, Group V, Melatonin group, Vitamin E group, Group VII and VIII did not show any significant change in brain AChE levels when compared to Group II (Table 10).

\section{DISCUSSION}

$\mathrm{DM}$ is an important risk factor for the development of cognitive impairment through several mechanisms. Further it has been documented that uncontrolled hyperglycaemia results in increased oxidative stress. ${ }^{14}$

The generation of oxygen free radicals (OFR) are supposed to be involved in the damage to endothelium, which is responsible for the progression of atherosclerosis. In addition to endothelial dysfunction, the central role of impaired lipid metabolism (lipid oxidation) is well established in the development of atherosclerosis. Advanced glycation end products (AGEs), formed as a result of prolonged hyperglycaemia, play a significant role in this process. One of the ways in which AGEs contribute to the development of atherosclerosis is by converting low-density lipoprotein (LDL) cholesterol to oxidized LDL (ox-LDL) cholesterol, which promotes its uptake by macrophages. This leads to the development of so-called "foam cells" and the subsequent formation of atherosclerotic plaques. Higher levels of oxidized cholesterol have been detected in patients with poorly controlled DM compared with those who achieve good glycaemic control. ${ }^{15}$

Increased formation of reactive oxygen species is an important feature of the diabetic endothelial cell dysfunction. This results in part from uncoupling of endothelial nitric oxide synthase such that it generates superoxide anion in addition to NO, forming peroxynitrite, a damaging molecule. This not only helps to explain the impairment of endothelial vasodilator mechanisms, but also increased progression of vascular disease. Many of these cellular abnormalities can be prevented by adequate scavenging of oxygen derived free radicals.

Cognitive dysfunction and dementia have recently been proven to be common (and under recognized) complications of diabetes mellitus (DM). In fact, several studies have demonstrated that alterations in glucose homeostasis increases risk for developing cognitive decline and dementia, including not only vascular dementia, but also Alzheimer's disease (AD). ${ }^{16}$

DM being an atherogenic risk factor, it may increase the risk of dementia. Decreased cholinergic transport across the blood-brain barrier observed in diabetic animals may exacerbate cognitive impairment. ${ }^{17}$

The actual mechanism of diabetes causing brain damage has not been completely elucidated but it appears to be a multifactorial process involving chronic metabolic and vascular disturbances resulting from fluctuations in blood 
glucose levels. ${ }^{18}$ It has been demonstrated that hyperglycaemia induces oxidative stress in various brain regions which perhaps results in cognitive impairment. ${ }^{19}$

In the current study melatonin, Vitamin E, Gliclazide, Atorvastatin, two plants-E. officinalis (Amla) and $M$. koenigii (Curry leaves) have been used to evaluate the antioxidant potential and their role in modulation of cognitive function. The study involved evaluation of various parameters like blood glucose level, lipid profile, malondialdehyde, reduced glutathione and acetylcholinesterase level in diabetic rats.

Fasting blood glucose levels and lipid profile were measured as the biochemical indicator of diabetes and hyperlipidaemia respectively. 30 days treatment of the diabetic rats with $E$. officinalis, $M$. koenigii and Gliclazide significantly decreased the blood glucose levels. The findings indicate that hyperglycaemia caused by STZ is significantly decreased by $E$. officinalis and $M$. koenigii and this effect is comparable to that of Gliclazide.

Diabetic control rats after 30 days showed changes in serum lipid profile. There was rise in serum total cholesterol, triglycerides and LDL cholesterol levels and fall of HDL cholesterol levels. In the present study $E$. officinalis $(500 \mathrm{mg} / \mathrm{kg})$, M. koenigii (250 and $500 \mathrm{mg} / \mathrm{kg}$ ) and Atorvastatin resulted in significant decrease in serum total cholesterol, triglycerides and LDL levels and increase in HDL level. Our results are in accordance with previous studies. $^{20,21}$

Transfer latency on elevated plus maze, step-down latency on passive avoidance apparatus and spatial navigation task in Morris-water maze were used to assess the behavioural parameters of learning and memory. Brain levels of MDA and GSH were measured as an analysis of oxidative stress and AChE levels in brain were measured as the biochemical parameter of cognition and memory. The transfer latency (TL) was significantly increased in both acquisition and retention components of TL in diabetic rats, suggestive of learning and memory impairment in these animals. 30 days treatment with high dose E. officinalis $(500 \mathrm{mg} / \mathrm{kg})$ significantly decreased the acquisition of TL, whereas E. officinalis $(500 \mathrm{mg} / \mathrm{kg})$, M. koenigii $(500 \mathrm{mg} / \mathrm{kg})$ and Melatonin significantly decreased the retention component of TL. The findings indicate that the learning and memory impairment in diabetic rats is significantly reversed and/or decreased by E. officinalis $(500 \mathrm{mg} / \mathrm{kg})$, M. koenigii $(500 \mathrm{mg} / \mathrm{kg})$ and this effect was comparable to that of Melatonin.

Acquisition and retention components of SDL was decreased significantly in diabetic rats, suggestive of learning and memory impairment. E. officinalis, $M$. koenigii, Melatonin, Gliclazide and Atorvastatin significantly increased acquisition and retention component, whereas Vitamin $\mathrm{E}$ increased retention component only. The above findings indicate that the learning and memory impairment caused by diabetes was significantly reversed and/or decreased by E. officinalis, $M$. koenigii and this effect was comparable to that of Melatonin, Gliclazide and Atorvastatin.

The spatial navigation task in Morris water maze was used to assess learning and memory behaviour, the test assessed two components of spatial navigation, viz. retention latency at day 15 and day 30 (RL-1 and RL-2 respectively) and time spent in target quadrant (QT-1 and QT-2 respectively). Findings indicates that diabetes significantly increased both RL-1 and RL-2, and significantly decreased both QT-1 and QT-2. RL-2 and QT-2 was reversed with all the drugs used in the study. The results indicate that the learning and memory impairment was significantly reversed and/or decreased by E. officinalis, $M$. koenigii and this effect was comparable to that of Melatonin, Vitamin E, Gliclazide and Atorvastatin. The above findings of behavioural tests were similar to other studies. ${ }^{18,22,23}$

The assessment of biochemical parameters of oxidative stress were done by measuring brain MDA and GSH levels. Diabetic rats showed significant increase in brain MDA and decrease in GSH level. E. officinalis, $M$. koenigii, Melatonin, Vitamin E and Gliclazide caused significant decrease in brain MDA and significant increase in brain GSH levels.

Diabetic rats showed significant increase in AChE activity whereas E. officinalis, M. koenigii and Melatonin significantly decreased the AChE activity. Earlier studies have shown the direct correlation between the AChE activity and cognitive dysfunction. ${ }^{24-26}$

The results of biochemical tests were in agreement with other studies which have verified the cognitive impairment in diabetic rats. ${ }^{26-28}$

In the present study, it was observed that E. officinalis and $M$. koenigii reversed and/or decreased the enhanced AChE activity (caused by diabetes) thus improving the learning and memory behaviours as indicated by behavioural tests conducted. The oxidative stress parameters (MDA and GSH) were also positively modulated by E. officinalis and M. koenigii in form of decreased oxidative damage to neurons.

This neuroprotective and neuro-modulatory effect of $E$. officinalis and $M$. koenigii resulting in improvement of learning and memory in diabetes might be exploited in diabetic patients with cognitive dysfunction. However further studies particularly clinical studies are required to determine the efficacy and safety of extract of $E$. officinalis and M. koenigii in diabetic patients who are suffering from cognitive dysfunction.

\section{CONCLUSION}

The results of the present study conclusively showed that both E. officinalis and $M$. koenigii have antioxidant activity in experimental diabetes mellitus. Both these 
drugs were effective in slowing the progression of cognitive impairment. The antioxidant activity of these drugs might be partially responsible for their effect in delaying cognitive impairment, besides this both the herbal extracts have got an anti-hyperglycaemic and hypolipidemic activity. However, future studies are required to provide an elaborate view on use of these two products in clinical medicine for treatment of diabetes and its neurological sequel.

Funding: No funding sources

Conflict of interest: None declared

Ethical approval: The study was approved by the Institutional Ethics Committee

\section{REFERENCES}

1. Ernest A, Peter S, Earl D. An update on the etiology and epidemiology on Diabetes mellitus. Ann N Y Acad Sci. 2006;1084:1-29.

2. Rao KB, AppaRao CH. Hypoglycemic and antihyperglycemic activity of alternifolium (Wt) Walp seed extract in normal and diabetic rats. Phytomed. 2001;8:88-93.

3. Upathaya V, Pandya K. Ayurvedic approach to diabetes mellitus and its management by indigenous resources, Bajaj JS (Ed.) Diabetes mellitus in developed countries. New delhi Inter Print. 1984;16:375-77.

4. Biessels GJ, Deary IJ, Ryan CM. Cognition and diabetes: a lifespan perspective. Lancet Neurol. 2008;7:184-90.

5. Jamwal KS, Sharma IP, Chopra CL. Pharmacological investigation on the fruits of Emblica officinalis. $\mathrm{J}$ Sci Ind Res. 1959;18:180-1.

6. Jayashri S, Jolly CI. Phytochemical, antibacterial and pharmacological investigations on Momordicachirantia and Emblica officinalis. Indian J Pharm Sci. 1993;1:6-13.

7. Achilya GS, Wadodkar SG, Dorle AK. Evaluation of hepatoprotective effect of Amalkadi Ghrita against carbon tetrachloride-induced hepatic damage in rats. J Ethnopharmacol. 2004;90:2-3.

8. Jose JK, Kuttan R. Hepatoprotective activity of Emblica officinalis and Chyavanaprash. J Ethnopharmacol. 2000;72:135-40.

9. Thakur CP, Mandal K. Effect of Emblica officinalis on cholesterol induced atherosclerosis in rabbits. $\mathbf{J}$ Med Res. 1984;79:142-6.

10. Vinuthan MK, Kumar GV, Ravindra JP, Narayana JK. Effect of extracts of Murraya koenigii leaves on the levels of blood glucose and plasma insulin in alloxan-induced diabetic rats. Indian J Physiol Pharmacol. 2004;48(3):348-52.

11. Ardura J, Gutierrez R, Andresn J, Agapito T. Emergence and evolution of the circadian rhythm of melatonin in children. Hormone Res. 2002;59:66-72.

12. Tan DX, Chen LD, Poeggeler B, Manchester LC, Reiter R. Melatonin: a potent, endogenous hydroxyl radical scavenger. Endocrine J. 1993;1:57-60.
13. Wright B, Sims D, Smart S, Alwazeer A, AldersonDay B, Allgar V, et al. Melatonin versus placebo in children with autism spectrum conditions and severe sleep problems not amenable to behaviour management strategies: a randomised controlled crossover trial. J Autism Dev Disorders. 2011;41:175-84.

14. Otsyula M, King MS, Ketcham TG, Sanders RA, Watkins JB. Oxidative stress in rats after 60 days of hypergalactosemia or hyperglycemia. Int $\mathrm{J}$ Toxicol. 2003;22:423-7.

15. Inouye M, Mio T, Sumino K. Link between glycation and lipoxidation in red blood cells in diabetes. Clin Chim Acta.1999;285:35-44.

16. S Roriz-Filho J, Sá-Roriz TM, Rosset I, Camozzato AL, Santos AC, Chaves ML, et al. (Pre)diabetes, brain aging, and cognition. Biochem Biophys Acta. 2009; 1792:432-43.

17. Pasquier F, Boulogne A, Leys D, Fontaine $P$. Diabetes mellitus and dementia. Diabetes Metab. 2006;32:403-14.

18. Manschot SM, Biessels GJ, Cameron NE, Cootter MA, Kamal A, Kappellle LJ, et al. Angiotensin converting enzyme inhibition partially prevents deficits in water maze performance, hippocampal synaptic plasticity and cerebral blood flow in streptozotocin-diabetic rats. Brain Res. 2003;966:274-82.

19. Tuzcu M, Baydas G. Effect of melatonin and vitamin $\mathrm{E}$ on diabetes-induced learning and memory impairment in rats. Eur J Pharmacol. 2006;537:10610.

20. Barbalho SM, Damasceno DC, Spada AP, Palhares M, Martuchi KA, Oshiiwa M, et al. Evaluation of glycemic and lipid profile of offspring of diabetic Wistar rats treated with Malpighia emarginata juice. Exp Diabetes Res. 2011;2011:173647.

21. Bhaskar VH, Ajay SS. Evaluation of antihperglycemic activity of extracts of Calotropis procera (Ait.) R.Br on streptozotocin induced diabetic rats. Global J Pharmacol. 2009;3:95-8.

22. Gispen WH, Biessels GJ. Cognition and synaptic plasticity in diabetes mellitus. Trends Neurosci. 2000;23:542-9.

23. Biessels GJ, Hiede LP, Kamal A. Ageing and diabetes: implications of brain function. Eur $\mathbf{J}$ Pharmacol. 2002;441:1-14.

24. Sarter M, Parikh V. Choline transporters, cholinergic transmission and cognition. Nat Rev Neurosci. 2005;6:48-56.

25. Sanchez-Chavez G, Salceda R. Effect of streptozotocin-induced diabetes on activities of cholinestereases in the rat retina. IUBMB life. 2000;49:283-7.

26. Schmatz R, Mazzanati CM, Spanevello R, Stefanello $\mathrm{N}$, Gutierres J, Correa M, et al. Resveratrol prevents memory deficits and the increase in acetylcholinesterase activity in streptozotocininduced diabetic rats. Eur J Pharmacol. 2009;610:428 . 
27. Kuhad A, Chopra K. Effect of sesamol on diabetesassociated cognitive decline in rats. Exp Brain Res. 2008;185:411-20.

28. Kuhad A, Chopra K. Curcumin attenuates diabetic encephalopathy in rats: behavioral and biochemical evidences. Eur J Pharmacol. 2007;576:34-42.
Cite this article as: Arora T, Kadali1 RP, Prasad DK. Evaluation of antioxidant potential of Emblica officinalis and Murraya koenigii and their role in modulation of cognitive function in diabetic rats. Int J Basic Clin Pharmacol 2019;8:2080-91. 